\title{
Fixed-Point Theorems in Complete Gauge Spaces and Applications to Second-Order Nonlinear Initial-Value Problems
}

\author{
Meryam Cherichi, ${ }^{1}$ Bessem Samet, ${ }^{2}$ and Calogero Vetro ${ }^{3}$ \\ ${ }^{1}$ Department of Mathematics, Faculty of Sciences of Tunis, University Campus, 2092 Tunis, Tunisia \\ ${ }^{2}$ Department of Mathematics, College of Science, King Saud University, P.O. Box 2455, Riyadh 11451, Saudi Arabia \\ ${ }^{3}$ Università degli Studi di Palermo, Dipartimento di Matematica e Informatica, Via Archirafi 34, 90123 Palermo, Italy
}

Correspondence should be addressed to Bessem Samet; bessem.samet@gmail.com

Received 3 May 2013; Accepted 10 August 2013

Academic Editor: Anna Kamińska

Copyright (C) 2013 Meryam Cherichi et al. This is an open access article distributed under the Creative Commons Attribution License, which permits unrestricted use, distribution, and reproduction in any medium, provided the original work is properly cited.

We establish fixed-point results for mappings and cyclic mappings satisfying a generalized contractive condition in a complete gauge space. Our theorems generalize and extend some fixed-point results in the literature. We apply our obtained results to the study of existence and uniqueness of solution to a second-order nonlinear initial-value problem.

\section{Introduction}

In the context of fixed-point theory, the metric fixed-point theory is the branch where metric conditions on the involved spaces and mappings play a crucial role in establishing theoretical results. In a certain sense, this theory is a farreaching outgrowth of a well-known theorem of Banach [1] which states that if $(X, d)$ is a complete metric space and if $T: X \rightarrow X$ is a mapping which satisfies the condition

$$
d(T x, T y) \leq k d(x, y), \quad \forall x, y \in X,
$$

where $0 \leq k<1$ is a constant, then $T$ has a unique fixed point. Since most of the spaces studied in mathematical analysis share many algebraic and topological properties as well as metric properties, there is no clear line separating the metric fixed-point theory from the topological or the settheoretic branch of the theory. Consequently, several authors considered the problem of existence (and uniqueness) of a fixed point for generalized contractions in a metric space (see, e.g., [2-9]). On the other hand, many definitions and theorems in the literature do not require that all of the properties of a metric hold true. For this reason, in the last decades, various concepts of generalized metrics were introduced (see, e.g., $[10,11]$ ). Here, we are interested in the so-called gauge spaces that are characterized by the fact that the distance between two points of the space may be zero even if the two points are distinct. For instance, Frigon [12] and Chiş and Precup [13] gave generalizations of the Banach contraction principle on gauge spaces (see also [14-16]). Consistent with this line of research, the aim of this paper is to present some fixed-point results for mappings and cyclic mappings satisfying a generalized contractive condition in a complete gauge space. Then, to illustrate the usefulness of our theorems, we apply our results to the study of existence and uniqueness of solutions to a second-order nonlinear initialvalue problem.

\section{Preliminaries}

In this section, we recall some preliminaries on gauge spaces and introduce some basic definitions.

Definition 1. Let $X$ be a nonempty set. A function $d: X \times$ $X \rightarrow[0,+\infty)$ is called a pseudometric in $X$ whenever the following hold:

(i) $d(x, x)=0$ for all $x \in X$,

(ii) $d(x, y)=d(y, x)$ for all $x, y \in X$,

(iii) $d(x, y) \leq d(x, z)+d(z, y)$ for all $x, y, z \in X$. 
Definition 2. Let $X$ be a nonempty set endowed with a pseudometric $d$. The $d$-ball of radius $\varepsilon>0$ centered at $x \in X$ is the set

$$
B(x ; d, \varepsilon)=\{y \in X \mid d(x, y)<\varepsilon\} .
$$

Definition 3. A family $\mathscr{F}=\left\{d_{\lambda} \mid \lambda \in \mathscr{A}\right\}$ of pseudometrics is called separating if, for each pair $(x, y)$ with $x \neq y$, there is a $d_{\lambda} \in \mathscr{F}$ such that $d_{\lambda}(x, y) \neq 0$.

Definition 4 . Let $X$ be a nonempty set, and let $\mathscr{F}=\left\{d_{\lambda} \mid\right.$ $\lambda \in \mathscr{A}\}$ be a separating family of pseudometrics on $X$. The topology $\mathscr{T}(\mathscr{F})$ having for a subbasis the family

$$
\mathscr{B}(\mathscr{F})=\left\{B\left(x ; d_{\lambda}, \varepsilon\right) \mid x \in X, d_{\lambda} \in \mathscr{F}, \varepsilon>0\right\}
$$

of balls is called the topology in $X$ induced by the family $\mathscr{F}$. The pair $(X, \mathscr{T}(\mathscr{F}))$ is called a gauge space. Note that $(X, \mathscr{T}(\mathscr{F}))$ is Hausdorff because we require $\mathscr{F}$ to be separating.

Definition 5. Let $(X, \mathscr{T}(\mathscr{F}))$ be a gauge space with respect to the family $\mathscr{F}=\left\{d_{\lambda} \mid \lambda \in \mathscr{A}\right\}$ of pseudometrics on $X$. Let $\left\{x_{n}\right\}$ be a sequence in $X$, and $x \in X$. Then, the following are considered.

(a) The sequence $\left\{x_{n}\right\}$ converges to $x$ if and only if

$$
\begin{aligned}
& \forall \lambda \in \mathscr{A}, \quad \forall \varepsilon>0, \quad \exists N \in \mathbb{N} \\
& \text { such that } d_{\lambda}\left(x_{n}, x\right)<\varepsilon, \quad \forall n \geq N .
\end{aligned}
$$

In this case, we denote that $x_{n} \stackrel{\mathscr{F}}{\rightarrow} x$.

(b) The sequence $\left\{x_{n}\right\}$ is Cauchy if and only if

$\forall \lambda \in \mathscr{A}, \quad \forall \varepsilon>0, \quad \exists N \in \mathbb{N}$

such that $d_{\lambda}\left(x_{n+p}, x_{n}\right)<\varepsilon, \quad \forall n \geq N, p \in \mathbb{N}$.

(c) $(X, \mathscr{T}(\mathscr{F}))$ is complete if and only if any Cauchy sequence in $(X, \mathscr{T}(\mathscr{F}))$ is convergent to an element of $X$.

(d) A subset of $X$ is said to be closed if it contains the limit of any convergent sequence of its elements.

For more details on gauge spaces, we refer the reader to [17]. Obviously, every metric space is automatically a pseudometric space. On the contrary, if a pseudometric $d$ is not a metric, it is because there are at least two points $x \neq y$ for which $d(x, y)=0$. In most situations, this does not happen; indeed, metrics come up in mathematics more often than pseudometrics. However, pseudometrics arise in a natural way in functional analysis and in the theory of hyperbolic complex manifolds [18].

\section{Main Results}

Let $\Psi$ denote the set of all functions $\psi:[0,+\infty) \rightarrow[0,+\infty)$ which satisfy the following conditions. (i) $\psi$ is continuous and nondecreasing.

(ii) $\psi(t)=0$ if and only if $t=0$.

Similarly, let $\Phi$ denote the set of all functions $\varphi$ : $[0,+\infty) \rightarrow[0,+\infty)$ which satisfy the following conditions.

(i) $\phi$ is lower semicontinuous.

(ii) $\phi(t)=0$ if and only if $t=0$.

Definition 6. Let $(X, \mathscr{T}(\mathscr{F}))$ be a gauge space, and let $T$ : $X \rightarrow X$ be a mapping. For $x \in X$, let $\mathscr{O}(x)$ denote the orbit of $x$; that is, $\mathcal{O}(x)=\left\{T^{n} x, n=0,1, \ldots\right\}$.

Our first result is the following theorem.

Theorem 7. Let $(X, \mathscr{T}(\mathscr{F}))$ be a complete gauge space, and let $T: X \rightarrow X$ be a mapping satisfying the following conditions.

(i) For all $\lambda \in \mathscr{A}$, for all $u \in X$, for all $x \in \mathcal{O}(u)$, and for all $y \in \overline{\mathcal{O}(u)}, d_{\lambda}(x, y)=0 \Rightarrow d_{\lambda}(T x, T y)=0$.

(ii) For all $\lambda \in \mathscr{A}$ and for all $0<a<b<\infty$, there exist $\psi_{\lambda, a, b} \in \Psi$ and $\varphi_{\lambda, a, b} \in \Phi$ such that

$$
\begin{aligned}
& \forall x, y \in X, \quad a \leq d_{\lambda}(x, y) \leq b \\
& \Longrightarrow \psi_{\lambda, a, b}\left(d_{\lambda}(T x, T y)\right) \\
& \quad \leq \psi_{\lambda, a, b}\left(d_{\lambda}(x, y)\right)-\varphi_{\lambda, a, b}\left(d_{\lambda}(x, y)\right) .
\end{aligned}
$$

Then, T has a unique fixed point.

Proof. First, we will remark that

$$
\begin{gathered}
\forall \lambda \in \mathscr{A}, \quad \forall x, y \in X, \quad d_{\lambda}(x, y) \neq 0 \\
\Longrightarrow d_{\lambda}(T x, T y)<d_{\lambda}(x, y) .
\end{gathered}
$$

In fact, let $\lambda \in \mathscr{A}$, and let $x, y \in X$ such that $d_{\lambda}(x, y) \neq 0$; by taking $a=d_{\lambda}(x, y)$ and $b=2 d_{\lambda}(x, y)$, we get, by applying assumption (ii) of the theorem, the existence of $\psi_{\lambda, a, b} \in \Psi$ and $\varphi_{\lambda, a, b} \in \Phi$ such that

$$
\psi_{\lambda, a, b}\left(d_{\lambda}(T x, T y)\right) \leq \psi_{\lambda, a, b}\left(d_{\lambda}(x, y)\right)-\varphi_{\lambda, a, b}\left(d_{\lambda}(x, y)\right) .
$$

Since $d_{\lambda}(x, y) \neq 0$, we have $\varphi_{\lambda, a, b}\left(d_{\lambda}(x, y)\right)>0$, and so we get

$$
\psi_{\lambda, a, b}\left(d_{\lambda}(T x, T y)\right)<\psi_{\lambda, a, b}\left(d_{\lambda}(x, y)\right) .
$$

Using the monotony of $\psi_{\lambda, a, b}$, we obtain the desired result (7). Moreover, assumption (i) implies that

$$
\begin{gathered}
\forall \lambda \in \mathscr{A}, \quad \forall u \in X, \quad \forall x \in \mathcal{O}(u), \quad \forall y \in \overline{\mathcal{O}(u)} \\
d_{\lambda}(T x, T y) \leq d_{\lambda}(x, y) .
\end{gathered}
$$

Now, let us take $x_{0} \in X$, and let $\left\{x_{n}\right\}$ be the sequence in $X$ given by

$$
x_{n+1}=T x_{n}, \quad \forall n \geq 0 .
$$

Thus, we complete the proof in the following four steps. 
Step 1. We will prove that

$$
d_{\lambda}\left(x_{n+1}, x_{n}\right) \longrightarrow 0 \quad \text { as } n \longrightarrow+\infty, \forall \lambda \in \mathscr{A}
$$

Let $\lambda \in \mathscr{A}$. Since $x_{n}, x_{n+1} \in \mathcal{O}\left(x_{0}\right)$, by applying (10), we obtain

$$
\begin{array}{r}
d_{\lambda}\left(x_{n+1}, x_{n}\right)=d_{\lambda}\left(T x_{n}, T x_{n-1}\right) \leq d_{\lambda}\left(x_{n}, x_{n-1}\right), \\
\forall n \geq 1 .
\end{array}
$$

It follows that $\left\{d_{\lambda}\left(x_{n+1}, x_{n}\right)\right\}$ is a decreasing sequence of nonnegative real numbers, and, hence, there is $r=r(\lambda) \geq 0$ such that

$$
d_{\lambda}\left(x_{n+1}, x_{n}\right) \longrightarrow r \text { as } n \longrightarrow+\infty
$$

Suppose that $r>0$; this implies that $r \leq d_{\lambda}\left(x_{n}, x_{n-1}\right) \leq$ $d_{\lambda}\left(x_{1}, x_{0}\right)$, for all $n \geq 1$. Now, taking $a=r / 2$ and $b=$ $d_{\lambda}\left(x_{1}, x_{0}\right)$, by applying (ii), we get the existence of $\psi_{\lambda, a, b} \in \Psi$ and $\varphi_{\lambda, a, b} \in \Phi$ such that

$$
\begin{aligned}
\psi_{\lambda, a, b}\left(d\left(x_{n+1}, x_{n}\right)\right) \leq & \psi_{\lambda, a, b}\left(d\left(x_{n}, x_{n-1}\right)\right) \\
& -\varphi_{\lambda, a, b}\left(d\left(x_{n}, x_{n-1}\right)\right), \quad \forall n \geq 1 .
\end{aligned}
$$

Letting $n \rightarrow+\infty$ in the above inequality, using the continuity of $\psi_{\lambda, a, b}$ and the lower semicontinuity of $\varphi_{\lambda, a, b}$, we get

$$
\psi_{\lambda, a, b}(r) \leq \psi_{\lambda, a, b}(r)-\varphi_{\lambda, a, b}(r)
$$

which implies that $r=0$. This is a contradiction, and, therefore, our claim (12) holds.

Step 2. We will prove that $\left\{x_{n}\right\}$ is a Cauchy sequence in $(X, \mathscr{T}(\mathscr{F}))$. Suppose that $\left\{x_{n}\right\}$ is not a Cauchy sequence. Then, there exists $(\lambda, \varepsilon) \in \mathscr{A} \times(0,+\infty)$ for which we can find two sequences of positive integers $\{m(k)\}$ and $\{n(k)\}$ such that, for all positive integers $k$,

$$
\begin{gathered}
n(k)>m(k)>k, \quad d_{\lambda}\left(x_{n(k)}, x_{m(k)}\right) \geq \varepsilon, \\
d_{\lambda}\left(x_{n(k)-1}, x_{m(k)}\right)<\varepsilon .
\end{gathered}
$$

Using (17) and the triangular inequality, we get

$$
\begin{aligned}
\varepsilon & \leq d_{\lambda}\left(x_{n(k)}, x_{m(k)}\right) \\
& \leq d_{\lambda}\left(x_{n(k)}, x_{n(k)-1}\right)+d_{\lambda}\left(x_{n(k)-1}, x_{m(k)}\right) \\
& <d_{\lambda}\left(x_{n(k)}, x_{n(k)-1}\right)+\varepsilon .
\end{aligned}
$$

Thus, we have

$$
\varepsilon \leq d_{\lambda}\left(x_{n(k)}, x_{m(k)}\right)<d\left(x_{n(k)}, x_{n(k)-1}\right)+\varepsilon .
$$

Letting $k \rightarrow+\infty$ in the above inequality and using (12), we obtain

$$
d_{\lambda}\left(x_{n(k)}, x_{m(k)}\right) \longrightarrow \varepsilon \quad \text { as } k \longrightarrow+\infty \text {. }
$$

By the triangular inequality, we have

$$
\begin{aligned}
& \left|d_{\lambda}\left(x_{n(k)+1}, x_{m(k)+1}\right)-d_{\lambda}\left(x_{n(k)}, x_{m(k)}\right)\right| \\
& \quad \leq d_{\lambda}\left(x_{n(k)+1}, x_{n(k)}\right)+d_{\lambda}\left(x_{m(k)}, x_{m(k)+1}\right) .
\end{aligned}
$$

Letting $k \rightarrow+\infty$ in the above inequality, using (12) and (20), we get

$$
d_{\lambda}\left(x_{n(k)+1}, x_{m(k)+1}\right) \longrightarrow \varepsilon \quad \text { as } k \longrightarrow+\infty .
$$

On the other hand, from (20), there exists a positive integer $k_{0}$ such that

$$
\frac{\varepsilon}{2} \leq d_{\lambda}\left(x_{n(k)}, x_{m(k)}\right) \leq 2 \varepsilon, \quad \forall k \geq k_{0} .
$$

Applying the hypothesis of the theorem by taking $a=\varepsilon / 2$ and $b=2 \varepsilon$, we get that there exist $\psi_{\lambda, a, b} \in \Psi$ and $\varphi_{\lambda, a, b} \in \Phi$ such that

$$
\begin{array}{r}
\psi_{\lambda, a, b}\left(d_{\lambda}\left(T x_{n(k)}, T x_{m(k)}\right)\right) \\
\leq \psi_{\lambda, a, b}\left(d_{\lambda}\left(x_{n(k)}, x_{m(k)}\right)\right)-\varphi_{\lambda, a, b}\left(d_{\lambda}\left(x_{n(k)}, x_{m(k)}\right)\right), \\
\forall k \geq k_{0} ;
\end{array}
$$

that is,

$$
\begin{array}{r}
\psi_{\lambda, a, b}\left(d_{\lambda}\left(x_{n(k)+1}, x_{m(k)+1}\right)\right) \\
\leq \psi_{\lambda, a, b}\left(d_{\lambda}\left(x_{n(k)}, x_{m(k)}\right)\right)-\varphi_{\lambda, a, b}\left(d_{\lambda}\left(x_{n(k)}, x_{m(k)}\right)\right), \\
\forall k \geq k_{0} .
\end{array}
$$

Letting $k \rightarrow+\infty$ in the above inequality, from (20), (22), the continuity of $\psi_{\lambda, a, b}$, and the lower semicontinuity of $\varphi_{\lambda, a, b}$, we obtain

$$
\psi_{\lambda, a, b}(\varepsilon) \leq \psi_{\lambda, a, b}(\varepsilon)-\varphi_{\lambda, a, b}(\varepsilon),
$$

which implies that $\varphi_{\lambda, a, b}(\varepsilon)=0$, which leads to the contradiction $\varepsilon=0$. Finally, we deduce that $\left\{x_{n}\right\}$ is a Cauchy sequence.

Step 3 (existence of fixed point). As $\left\{x_{n}\right\}$ is a Cauchy sequence in the complete gauge space $(X, \mathscr{T}(\mathscr{F}))$, then there exists $x^{*} \in$ $X$ such that

$$
x_{n} \stackrel{\mathscr{F}}{\longrightarrow} x^{*} \quad \text { as } n \longrightarrow+\infty \text {. }
$$

This implies that $x^{*} \in \overline{\mathcal{O}\left(x_{0}\right)}$. On the other hand, by (10), for all $x_{n} \in \mathcal{O}\left(x_{0}\right)$, we get

$$
d_{\lambda}\left(T x_{n}, T x^{*}\right) \leq d_{\lambda}\left(x_{n}, x^{*}\right), \quad \forall \lambda \in \mathscr{A}, \forall n \geq 0 .
$$

Now, from

$$
\begin{aligned}
d_{\lambda}\left(x^{*}, T x^{*}\right) & \leq d_{\lambda}\left(x^{*}, x_{n+1}\right)+d_{\lambda}\left(T x_{n}, T x^{*}\right) \\
& \leq d_{\lambda}\left(x^{*}, x_{n+1}\right)+d_{\lambda}\left(x_{n}, x^{*}\right)
\end{aligned}
$$


for all $\lambda \in \mathscr{A}$, letting $n \rightarrow+\infty$, we obtain $d_{\lambda}\left(x^{*}, T x^{*}\right) \leq 0$, for all $\lambda \in \mathscr{A}$.

In the virtue of the separating structure of $\mathscr{F}$, we deduce that $T x^{*}=x^{*}$, and, hence, the existence of the fixed point is proved.

Step 4 (uniqueness). Suppose that there exist $x^{*}, y^{*}$ such that $x^{*} \neq y^{*}, T x^{*}=x^{*}$, and $T y^{*}=y^{*}$. Then, there exists $\lambda \in$ $\mathscr{A}$ such that $d_{\lambda}\left(x^{*}, y^{*}\right) \neq 0$, and so, by using (7), we get a contradiction.

Remark 8. Theorem 7 is a generalization of the main result of [6].

By taking $\psi_{\lambda, a, b}(t)=t$ in Theorem 7 , we get the following corollary.

Corollary 9. Let $(X, \mathscr{T}(\mathscr{F}))$ be a complete gauge space, and let $T: X \rightarrow X$ be a mapping satisfying the following conditions.

(i) For all $\lambda \in \mathscr{A}$, for all $u \in X$, for all $x \in \mathcal{O}(u)$, and for all $y \in \overline{\mathcal{O}(u)}, d_{\lambda}(x, y)=0 \Rightarrow d_{\lambda}(T x, T y)=0$.

(ii) For all $\lambda \in \mathscr{A}$ and for all $0<a<b<+\infty$, there exists $\varphi_{\lambda, a, b} \in \Phi$ such that

$$
\begin{aligned}
& \forall x, y \in X, \quad a \leq d_{\lambda}(x, y) \leq b \\
& \quad \Longrightarrow d_{\lambda}(T x, T y) \leq d_{\lambda}(x, y)-\varphi_{\lambda, a, b}\left(d_{\lambda}(x, y)\right) .
\end{aligned}
$$

Then, $T$ has a unique fixed point.

By taking $\varphi_{\lambda, a, b}(t)=(1-k) t$ in Corollary 9 , we get the following result.

Corollary 10. Let $(X, \mathscr{T}(\mathscr{F}))$ be a complete gauge space, and let $T: X \rightarrow X$ be a mapping satisfying the following conditions.

(i) For all $\lambda \in \mathscr{A}$, for all $u \in X$, for all $x \in \mathcal{O}(u)$, and for all $y \in \overline{\mathcal{O}(u)}, d_{\lambda}(x, y)=0 \Rightarrow d_{\lambda}(T x, T y)=0$.

(ii) For all $\lambda \in \mathscr{A}$ and for all $0<a<b<\infty$, there exists $k=k(\lambda, a, b) \in(0,1)$ such that

$$
\begin{aligned}
& \forall x, y \in X, \quad a \leq d_{\lambda}(x, y) \leq b \\
& \Longrightarrow d_{\lambda}(T x, T y) \leq k d_{\lambda}(x, y) .
\end{aligned}
$$

Then, T has a unique fixed point.

Next, we give other consequences of Theorem 7.

Corollary 11. Let $(X, \mathscr{T}(\mathscr{F}))$ be a complete gauge space, and let $T: X \rightarrow X$ be a mapping satisfying the following condition.

For all $\lambda \in \mathscr{A}$, there exist $\psi_{\lambda} \in \Psi$ and $\varphi_{\lambda} \in \Phi$ such that

$$
\begin{array}{r}
\psi_{\lambda}\left(d_{\lambda}(T x, T y)\right) \leq \psi_{\lambda}\left(d_{\lambda}(x, y)\right)-\varphi_{\lambda}\left(d_{\lambda}(x, y)\right) \\
\forall x, y \in X .
\end{array}
$$

Then, T has a unique fixed point.

Proof. Condition (i) of Theorem 7 is satisfied from inequality (32), and taking $\psi_{\lambda, a, b}(t)=\psi_{\lambda}(t)$ and $\varphi_{\lambda, a, b}(t)=\varphi_{\lambda}(t)$, we get Corollary 11.
By taking $\psi_{\lambda}(t)=t$ in Corollary 11, we get the following result.

Corollary 12. Let $(X, \mathscr{T}(\mathscr{F}))$ be a complete gauge space, and let $T: X \rightarrow X$ be a mapping satisfying the following condition.

For all $\lambda \in \mathscr{A}$, there exists $\varphi_{\lambda} \in \Phi$ such that

$$
\forall x, y \in X, \quad d_{\lambda}(T x, T y) \leq d_{\lambda}(x, y)-\varphi_{\lambda}\left(d_{\lambda}(x, y)\right) .
$$

Then, T has a unique fixed point.

Also, by taking $\varphi_{\lambda}(t)=(1-k) t$ in Corollary 12 , we get the following result.

Corollary 13. Let $(X, \mathscr{T}(\mathscr{F}))$ be a complete gauge space, and let $T: X \rightarrow X$ be a mapping satisfying the following condition.

For all $\lambda \in \mathscr{A}$, there exists $k=k(\lambda) \in(0,1)$ such that

$$
\forall x, y \in X, \quad d_{\lambda}(T x, T y) \leq k d_{\lambda}(x, y) .
$$

Then, T has a unique fixed point.

Finally, we give a result involving a condition of integral type. Let $\Gamma$ denote the set of all functions $\alpha:[0,+\infty) \rightarrow$ $[0,+\infty)$ which satisfy the following conditions.

(c1) $\alpha$ is Lebesgue integrable on $[0,+\infty)$.

(c2) For all $\varepsilon>0$, we have $\int_{0}^{\varepsilon} \alpha(t) d t>0$.

Corollary 14. Let $(X, \mathscr{T}(\mathscr{F}))$ be a complete gauge space, and let $T: X \rightarrow X$ be a mapping satisfying the following conditions.

(i) For all $\lambda \in \mathscr{A}$, for all $u \in X$, for all $x \in \mathcal{O}(u)$, and for all $y \in \overline{\mathcal{O}(u)}, d_{\lambda}(x, y)=0 \Rightarrow d_{\lambda}(T x, T y)=0$.

(ii) For all $0<a<b<\infty$ and for all $\lambda \in \mathscr{A}$, there exist $\alpha_{\lambda, a, b}, \beta_{\lambda, a, b} \in \Gamma$ such that

$$
\begin{aligned}
\forall x, y \in X, \quad a \leq d_{\lambda}(x, y) \leq b \\
\quad \Longrightarrow \int_{0}^{d_{\lambda}(T x, T y)} \alpha_{\lambda, a, b}(t) d t \\
\quad \leq \int_{0}^{d_{\lambda}(x, y)} \alpha_{\lambda, a, b}(t) d t-\int_{0}^{d_{\lambda}(x, y)} \beta_{\lambda, a, b}(t) d t .
\end{aligned}
$$

Then, T has a unique fixed point.

Proof. It follows from Theorem 7 that, by taking

$$
\begin{gathered}
\psi_{\lambda, a, b}(x)=\int_{0}^{x} \alpha_{\lambda, a, b}(t) d t \\
\varphi_{\lambda, a, b}(x)=\int_{0}^{x} \beta_{\lambda, a, b}(t) d t, \quad x \geq 0 .
\end{gathered}
$$

Remark 15. Corollary 14 is a generalization of the main result of [19]. 


\section{Results for Cyclic Mappings}

In [20], Kirk et al. obtained extensions of the Banach contraction principle for cyclic mappings, by considering a cyclical contractive condition as given by the following theorem.

Definition 16. Let $\left\{A_{i}\right\}_{i=1}^{p}$ be nonempty subsets of a nonempty set $X$. Then, $T: X \rightarrow X$ is called a cyclic mapping associated with $\left(A_{1}, \ldots, A_{p}\right)$ if the following conditions hold:

(i) $X=\bigcup_{i=1}^{p} A_{i}$,

(ii) $T\left(A_{i}\right) \subseteq A_{i+1}$ for $1 \leq i \leq p$, where $A_{p+1}=A_{1}$.

Theorem 17. Let $\left\{A_{i}\right\}_{i=1}^{p}$ be nonempty closed subsets of a metric space $(X, d)$, and suppose that $T: X \rightarrow X$ is a cyclic mapping associated with $\left(A_{1}, \ldots, A_{p}\right)$ satisfying the following:

$\exists k \in(0,1) \quad$ such that $d(T x, T y) \leq k d(x, y)$,

$$
\forall x \in A_{i}, \quad y \in A_{i+1}, \quad \text { for } 1 \leq i \leq p .
$$

Then, $T$ has a unique fixed point.

Inspired by this result, other fixed-point theorems with cyclical contractive conditions were obtained (see [21-30]). Our aim in this section is to extend Theorem 7 for cyclic mappings.

Theorem 18. Let $\left\{A_{i}\right\}_{i=1}^{p}$ be nonempty closed subsets of a complete gauge space $(X, \mathscr{T}(\mathscr{F}))$, and suppose that $T: X \rightarrow$ $X$ is a cyclic mapping associated with $\left(A_{1}, \ldots, A_{p}\right)$ satisfying the following conditions.

(i) For all $\lambda \in \mathscr{A}$, for all $u \in X$, for $1 \leq i \leq p$, and for all $x \in \mathcal{O}(u) \bigcap A_{i}, y \in \overline{\mathcal{O}(u)} \bigcap A_{i+1}$,

$$
d_{\lambda}(x, y)=0 \Longrightarrow d_{\lambda}(T x, T y)=0 .
$$

(ii) For all $\lambda \in \mathscr{A}$ and for all $0<a<b<\infty$, there exist $\psi_{\lambda, a, b} \in \Psi$ and $\varphi_{\lambda, a, b} \in \Phi$ such that, for $1 \leq i \leq p$,

$$
\begin{aligned}
\forall x \in A_{i}, \quad y \in A_{i+1}, \quad a \leq d_{\lambda}(x, y) \leq b \\
\Longrightarrow \psi_{\lambda, a, b}\left(d_{\lambda}(T x, T y)\right) \\
\quad \leq \psi_{\lambda, a, b}\left(d_{\lambda}(x, y)\right)-\varphi_{\lambda, a, b}\left(d_{\lambda}(x, y)\right) .
\end{aligned}
$$

Then, T has a unique fixed point.

Proof. As in the proof of Theorem 7, one can prove that

$$
\begin{aligned}
& \forall \lambda \in \mathscr{A}, \quad \forall x \in A_{i}, \quad y \in A_{i+1}, \\
& d_{\lambda}(x, y) \neq 0 \Longrightarrow d_{\lambda}(T x, T y)<d_{\lambda}(x, y), \\
& \forall \lambda \in \mathscr{A}, \quad \forall u \in X, \quad \forall x \in \mathcal{O}(u) \cap A_{i}, \\
& y \in \overline{\mathcal{O}(u)} \cap A_{i+1}, \quad d_{\lambda}(T x, T y) \leq d_{\lambda}(x, y) .
\end{aligned}
$$

Next, in establishing the existence of a fixed point, since $T\left(\bigcap_{i=1}^{p} A_{i}\right) \subset \bigcap_{i=1}^{p} A_{i}$, one only needs to prove that $\bigcap_{i=1}^{p} A_{i} \neq \emptyset$. In fact, applying Theorem 7 to the restriction of $T$ on the complete gauge space $\left(\bigcap_{i=1}^{p} A_{i}, \mathscr{T}(\mathscr{F})\right)$, we obtain that $T$ has a fixed point in $\bigcap_{i=1}^{p} A_{i}$.

Now, let $x_{0} \in A_{1}$, and we construct a sequence $\left\{x_{n}\right\}$ in $X$ such that

$$
x_{n+1}=T x_{n}, \quad \forall n \geq 0 .
$$

Remark that, for all $n \geq 0$, there exists $i=i(n) \in\{1, \ldots, p\}$ such that $\left(x_{n}, x_{n+1}\right) \in A_{i} \times A_{i+1}$. Then, as in the proof of Theorem 7 , we obtain that

$$
d_{\lambda}\left(x_{n-1}, x_{n}\right) \longrightarrow 0 \quad \text { as } n \longrightarrow+\infty .
$$

We will prove that $\left\{x_{n}\right\}$ is a Cauchy sequence in the complete gauge space $(X, \mathscr{T}(\mathscr{F}))$. Suppose that $\left\{x_{n}\right\}$ is not a Cauchy sequence. Then, there exists $(\lambda, \varepsilon) \in \mathscr{A} \times(0,+\infty)$ for which we can find two sequences of positive integers $\{m(k)\}$ and $\{n(k)\}$ such that, for all positive integers $k$,

$$
\begin{gathered}
n(k)>m(k)>k, \quad d_{\lambda}\left(x_{m(k)}, x_{n(k)}\right) \geq \varepsilon, \\
d_{\lambda}\left(x_{m(k)}, x_{n(k)-1}\right)<\varepsilon .
\end{gathered}
$$

Using (44) and the triangular inequality, we get

$$
\begin{aligned}
\varepsilon & \leq d_{\lambda}\left(x_{n(k)}, x_{m(k)}\right) \\
& \leq d_{\lambda}\left(x_{m(k)}, x_{n(k)-1}\right)+d_{\lambda}\left(x_{n(k)-1}, x_{n(k)}\right) \\
& <\varepsilon+d_{\lambda}\left(x_{n(k)}, x_{n(k)-1}\right) .
\end{aligned}
$$

Thus, we have

$$
\varepsilon \leq d_{\lambda}\left(x_{n(k)}, x_{m(k)}\right)<\varepsilon+d_{\lambda}\left(x_{n(k)}, x_{n(k)-1}\right) .
$$

Letting $k \rightarrow+\infty$ in the above inequality and using (43), we obtain

$$
d_{\lambda}\left(x_{n(k)}, x_{m(k)}\right) \longrightarrow \varepsilon \text { as } k \longrightarrow+\infty .
$$

Let $k$ be a positive integer, and let $j(k) \in\{1, \ldots, p\}$ be the integer such that

$$
n(k)-m(k)=j(k) \quad \bmod p .
$$

Using the triangular inequality, we have

$$
\begin{aligned}
d_{\lambda}\left(x_{n(k)}, x_{m(k)}\right) \leq & d_{\lambda}\left(x_{n(k)}, x_{n(k)+1}\right) \\
& +d_{\lambda}\left(x_{n(k)+1}, x_{m(k)+j(k)}\right) \\
& +\sum_{i=0}^{j(k)-1} d_{\lambda}\left(x_{m(k)+1+i}, x_{m(k)+i}\right), \\
d_{\lambda}\left(x_{n(k)+1}, x_{m(k)+j(k)}\right) \leq & d_{\lambda}\left(x_{n(k)+1}, x_{n(k)}\right) \\
& +d_{\lambda}\left(x_{n(k)}, x_{m(k)}\right) \\
& +\sum_{i=0}^{j(k)-1} d_{\lambda}\left(x_{m(k)+1+i}, x_{m(k)+i}\right) .
\end{aligned}
$$


Thus, we have

$$
\begin{aligned}
& \left|d_{\lambda}\left(x_{n(k)+1}, x_{m(k)+j(k)}\right)-d_{\lambda}\left(x_{n(k)}, x_{m(k)}\right)\right| \\
& \leq d_{\lambda}\left(x_{n(k)+1}, x_{n(k)}\right)+\sum_{i=0}^{j(k)-1} d_{\lambda}\left(x_{m(k)+1+i}, x_{m(k)+i}\right) \\
& \quad \leq d_{\lambda}\left(x_{n(k)+1}, x_{n(k)}\right)+\sum_{i=0}^{p-1} d_{\lambda}\left(x_{m(k)+1+i}, x_{m(k)+i}\right) .
\end{aligned}
$$

Letting $k \rightarrow+\infty$ in the above inequality, using (43) and (47), we get that

$$
d_{\lambda}\left(x_{n(k)+1}, x_{m(k)+j(k)}\right) \longrightarrow \varepsilon \text { as } k \longrightarrow+\infty .
$$

Using the same technique, we obtain that

$$
d_{\lambda}\left(x_{n(k)}, x_{m(k)+j(k)-1}\right) \longrightarrow \varepsilon \quad \text { as } k \longrightarrow+\infty .
$$

On the other hand, from (52), there exists a positive integer $k_{0}$ such that

$$
\frac{\varepsilon}{2} \leq d_{\lambda}\left(x_{n(k)}, x_{m(k)+j(k)-1}\right) \leq 2 \varepsilon, \quad \forall k \geq k_{0} .
$$

Applying the hypothesis of the theorem by taking $a=\varepsilon / 2$ and $b=2 \varepsilon$, we get the existence of $\psi_{\lambda, a, b} \in \Psi$ and $\varphi_{\lambda, a, b} \in \Phi$ such that

$$
\begin{aligned}
\psi_{\lambda, a, b} & \left(d_{\lambda}\left(T x_{n(k)}, T x_{m(k)+j(k)-1}\right)\right) \\
\leq & \psi_{\lambda, a, b}\left(d_{\lambda}\left(x_{n(k)}, x_{m(k)+j(k)-1}\right)\right) \\
& -\varphi_{\lambda, a, b}\left(d_{\lambda}\left(x_{n(k)}, x_{m(k)+j(k)-1}\right)\right) ;
\end{aligned}
$$

that is,

$$
\begin{aligned}
\psi_{\lambda, a, b} & \left(d_{\lambda}\left(x_{n(k)+1}, x_{m(k)+j(k)}\right)\right) \\
\leq & \psi_{\lambda, a, b}\left(d_{\lambda}\left(x_{n(k)}, x_{m(k)+j(k)-1}\right)\right) \\
& -\varphi_{\lambda, a, b}\left(d_{\lambda}\left(x_{n(k)}, x_{m(k)+j(k)-1}\right)\right) .
\end{aligned}
$$

Letting $k \rightarrow+\infty$ in the above inequality, using (51), (52), the continuity of $\psi_{\lambda, a, b}$, and the lower semicontinuity of $\varphi_{\lambda, a, b}$, we obtain

$$
\psi_{\lambda, a, b}(\varepsilon) \leq \psi_{\lambda, a, b}(\varepsilon)-\varphi_{\lambda, a, b}(\varepsilon),
$$

which implies that $\varphi_{\lambda, a, b}(\varepsilon)=0$, which leads to the contradiction $\varepsilon=0$. Finally, we deduce that $\left\{x_{n}\right\}$ is a Cauchy sequence in the complete gauge space $(X, \mathscr{T}(\mathscr{F}))$; therefore, $\left\{x_{n}\right\}$ converges to some $x^{*} \in X$. In view of condition (ii) of Definition 16, an infinite number of elements of the sequence $\left\{x_{n}\right\}$ lie in each $A_{i}, i \in\{1, \ldots, p\}$. As $A_{i}$ is a closed subset, we deduce that $x^{*} \in A_{i}$, for $i \in\{1, \ldots, p\}$. Thus, $x^{*} \in \bigcap_{i=1}^{p} A_{i}$, and so $\bigcap_{i=1}^{p} A_{i} \neq \emptyset$.

In order to complete the proof, we have to show that uniqueness of the fixed point.

From condition (ii) of Definition 16, any fixed point of $T$ lies necessarily in $\bigcap_{i=1}^{p} A_{i}$. Suppose that there exist $x^{*}, y^{*} \in X$ such that $x^{*} \neq y^{*}, T x^{*}=x^{*}$, and $T y^{*}=y^{*}$. Then, there exists $\lambda \in \mathscr{A}$ such that $d_{\lambda}\left(x^{*}, y^{*}\right) \neq 0$, and so, by using (40), we get a contradiction.
Remark 19. Theorem 18 is a generalization of the main result of [24].

By taking $\psi_{\lambda, a, b}(t)=t$ in Theorem 18, we get the following corollary.

Corollary 20. Let $\left\{A_{i}\right\}_{i=1}^{p}$ be nonempty closed subsets of a complete gauge space $(X, \mathscr{T}(\mathscr{F}))$, and suppose that $T: X \rightarrow$ $X$ is a cyclic mapping associated with $\left(A_{1}, \ldots, A_{p}\right)$ satisfying the following conditions.

(i) For all $\lambda \in \mathscr{A}$, for all $u \in X$, for $1 \leq i \leq p$, and for all $x \in \mathcal{O}(u) \bigcap A_{i}, y \in \overline{\mathcal{O}(u)} \bigcap A_{i+1}$,

$$
d_{\lambda}(x, y)=0 \Longrightarrow d_{\lambda}(T x, T y)=0 \text {. }
$$

(ii) For all $\lambda \in \mathscr{A}$ and for all $0<a<b<\infty$, there exists $\varphi_{\lambda, a, b} \in \Phi$ such that, for $1 \leq i \leq p$,

$$
\begin{gathered}
\forall x \in A_{i}, \quad y \in A_{i+1}, \\
a \leq d_{\lambda}(x, y) \leq b \\
\Longrightarrow d_{\lambda}(T x, T y) \leq d_{\lambda}(x, y)-\varphi_{\lambda, a, b}\left(d_{\lambda}(x, y)\right) .
\end{gathered}
$$

Then, $T$ has a unique fixed point.

The analogous of Corollaries 10-14 for cyclic mappings can be obtained easily, and so, to avoid repetitions, we omit the details.

\section{Application to Ordinary Differential Equation}

In this section, we present an example where our obtained results can be applied. Precisely, we study the existence of solution for the following second-order nonlinear initialvalue problem:

$$
\begin{gathered}
x^{\prime \prime}(t)= \\
k(t, x(t)), \quad t>0, \\
x(0)=\alpha, \\
x^{\prime}(0)=\beta,
\end{gathered}
$$

where $k:[0,+\infty) \times \mathbb{R}^{n} \rightarrow \mathbb{R}^{n}$ is a continuous function. This problem is equivalent to the integral equation

$$
x(t)=\int_{0}^{t}(t-s) k(s, x(s)) d s+\beta t+\alpha, \quad t \geq 0 .
$$

Denote by $X=C\left([0,+\infty), \mathbb{R}^{n}\right)$ the set of continuous functions defined on $[0,+\infty)$. For each positive integer $p \geq 1$, we define the function $\|\cdot\|_{p}: X \rightarrow[0,+\infty)$ by

$$
\|x\|_{p}=\max _{0 \leq t \leq p}|x(t)|, \quad \forall x \in X .
$$

This function is a seminorm on $X$. Also, define

$$
d_{p}(x, y)=\|x-y\|_{p}, \quad \forall p \geq 1, x, y \in X .
$$

Then, $\mathscr{F}=\left\{d_{p}\right\}_{p \geq 1}$ is a separating family of pseudometrics on $X$, and $(X, \mathscr{T}(\mathscr{F}))$ is a complete gauge space. 
We will prove the following result.

Theorem 21. Suppose that, for all $0<a<b<\infty$, for each $s \geq 0$, and for all $x, y \in X$, one has

$$
\begin{aligned}
a<d_{p}(x, y)<b \Longrightarrow & |k(s, x(s))-k(s, y(s))| \\
& \leq \gamma(s) \frac{(b-a) d_{p}(x, y)^{2}}{1+(b-a) d_{p}(x, y)},
\end{aligned}
$$

where $\gamma:[0,+\infty) \rightarrow[0,+\infty)$ is such that the function $t \mapsto$ $\int_{0}^{t}(t-s) \gamma(s) d s$ is bounded on $[0,+\infty)$ and

$$
\sup _{t \geq 0} \int_{0}^{t}(t-s) \gamma(s) d s \leq 1 .
$$

Then, (59) has a unique solution $x^{*} \in C\left([0,+\infty), \mathbb{R}^{n}\right)$.

Proof. Consider the operator $T: X \rightarrow X$ given by

$$
\begin{array}{r}
T x(t)=\int_{0}^{t}(t-s) k(s, x(s)) d s+\beta t+\alpha, \\
t \geq 0, \quad x \in X .
\end{array}
$$

Clearly, $T$ is well defined since $k$ is a continuous function.

Now, $x^{*}$ is a solution of (59) if and only if $x^{*}$ is a fixed point of $T$. Next, we will show that the two conditions of Corollary 9 hold true.

Condition (i). Let $p$ be a positive integer such that $p \geq 1$, and let $x, y \in X$ such that $d_{p}(x, y)=0$. This means that

$$
y(s)=x(s), \quad \forall s \in[0, p] .
$$

Let us take $t \in[0, p]$; then, we have

$$
\begin{aligned}
& T y(t)-T x(t) \\
&=\int_{0}^{t}(t-s)(k(s, y(s))-k(s, x(s))) d s=0 .
\end{aligned}
$$

Hence,

$$
d_{p}(T x, T y)=\max _{0 \leq t \leq p}|T y(t)-T x(t)|=0 .
$$

Thus, the condition is satisfied.

Condition (ii). Let $a, b \in \mathbb{R}$ such that $0<a<b$, and let $x, y \in X$ such that $a<d_{p}(x, y)<b$. For all $t \in[0, p], p \geq 1$, we have

$$
\begin{aligned}
|T x(t)-T y(t)| & \leq \int_{0}^{t}(t-s)|k(s, y(s))-k(s, x(s))| d s \\
& \leq \int_{0}^{t}(t-s) \gamma(s) \frac{(b-a) d_{p}(x, y)^{2}}{1+(b-a) d_{p}(x, y)} d s \\
& \leq \frac{(b-a) d_{p}(x, y)^{2}}{1+(b-a) d_{p}(x, y)} \int_{0}^{t}(t-s) \gamma(s) d s \\
& \leq \frac{(b-a) d_{p}(x, y)^{2}}{1+(b-a) d_{p}(x, y)} .
\end{aligned}
$$

Then, for all $p \geq 1$, we have

$$
d_{p}(T x, T y) \leq \frac{(b-a) d_{p}(x, y)^{2}}{1+(b-a) d_{p}(x, y)}
$$

Hence, for all $p \geq 1$, for all $0<a<b<\infty$, and for all $x, y \in X$ such that $a<d_{p}(x, y)<b$, we have

$$
d_{p}(T x, T y) \leq d_{p}(x, y)-\varphi_{p, a, b}\left(d_{p}(x, y)\right)
$$

where $\varphi_{p, a, b}(t)=t-(b-a) t^{2} /(1+(b-a) t)$, and, obviously, $\varphi_{p, a, b} \in \Phi$. Finally, applying Corollary 9 , we obtain that $T$ has a unique fixed point $x^{*} \in X$; that is, $x^{*} \in C\left([0,+\infty), \mathbb{R}^{n}\right)$ is the unique solution of (59).

\section{Acknowledgment}

The authors would like to extend their sincere appreciation to the Deanship of Scientific Research at King Saud University for the funding of this research through the Research Group Project no. RGP-VPP-237.

\section{References}

[1] S. Banach, "Sur les opèrations dans les ensembles abstraits et leur application aux equations intègrales," Fundamenta Mathematicae, vol. 3, pp. 133-181, 1922.

[2] R. P. Agarwal, M. Meehan, and D. O'Regan, Fixed Point Theory and Applications, vol. 141, Cambridge University Press, Cambridge, UK, 2001.

[3] Ya. I. Alber and S. Guerre-Delabriere, "Principle of weakly contractive maps in Hilbert spaces," in New Results in Operator Theory and Its Applications, I. Gohberg and Y. I. Lyubich, Eds., vol. 98 of Operator Theory: Advances and Applications, pp. 7-22, Birkhäuser, Basel, Switzerland, 1997.

[4] L. J. Ćirić, "A generalization of Banach's contraction principle," Proceedings of the American Mathematical Society, vol. 45, pp. 267-273, 1974.

[5] L. J. Ćirić, "Non-self mappings satisfying non-linear contractive condition with applications," Nonlinear Analysis. Theory, Methods \& Applications, vol. 71, no. 7-8, pp. 2927-2935, 2009.

[6] P. N. Dutta and B. S. Choudhury, "A generalisation of contraction principle in metric spaces," Fixed Point Theory and Applications, vol. 2008, Article ID 406368, 8 pages, 2008.

[7] M. S. Khan, M. Swaleh, and S. Sessa, "Fixed point theorems by altering distances between the points," Bulletin of the Australian Mathematical Society, vol. 30, no. 1, pp. 1-9, 1984.

[8] B. E. Rhoades, "Some theorems on weakly contractive maps," Nonlinear Analysis. Theory, Methods \& Applications, vol. 47, no. 4, pp. 2683-2693, 2001.

[9] E. Zeidler, Nonlinear Functional Analysis and its Applications I: Fixed Point Theorems, Springer, Berlin, Germany, 1986.

[10] R. C. Freiwald, Introduction To Set Theory and Topology, Lecture Notes, Washington University, St. Louis, Mo, USA, 2010.

[11] M. B. Smyth, Topology and Category Theory in Computer Science, Oxford University, New York, NY, USA, 1991.

[12] M. Frigon, "Fixed point results for generalized contractions in gauge spaces and applications," Proceedings of the American Mathematical Society, vol. 128, no. 10, pp. 2957-2965, 2000. 
[13] A. Chiş and R. Precup, "Continuation theory for general contractions in gauge spaces," Fixed Point Theory and Applications, vol. 3, pp. 173-185, 2004.

[14] R. P. Agarwal and D. O'Regan, "Fixed-point theorems for multivalued maps with closed values on complete gauge spaces," Applied Mathematics Letters, vol. 14, no. 7, pp. 831-836, 2001.

[15] M. Cherichi and B. Samet, "Fixed point theorems on ordered gauge spaces with applications to nonlinear integral equations," Fixed Point Theory and Applications, vol. 2012, article 13, 2012.

[16] C. Chifu and G. Petruşel, "Fixed-point results for generalized contractions on ordered gauge spaces with applications," Fixed Point Theory and Applications, vol. 2011, Article ID 979586, 10 pages, 2011.

[17] J. Dugundji, Topology, Allyn and Bacon, Boston, Mass, USA, 1966.

[18] S. Kobayashi, "Intrinsic metrics on complex manifolds," Bulletin of the American Mathematical Society, vol. 73, pp. 347-349, 1967.

[19] A. Branciari, "A fixed point theorem for mappings satisfying a general contractive condition of integral type," International Journal of Mathematics and Mathematical Sciences, vol. 29, no. 9, pp. 531-536, 2002.

[20] W. A. Kirk, P. S. Srinivasan, and P. Veeramani, "Fixed points for mappings satisfying cyclical contractive conditions," Fixed Point Theory, vol. 4, no. 1, pp. 79-89, 2003.

[21] R. P. Agarwal, M. A. Alghamdi, and N. Shahzad, "Fixed point theory for cyclic generalized contractions in partial metric spaces," Fixed Point Theory and Applications, vol. 2012, article 40, 2012.

[22] M. Jleli, E. Karapınar, and B. Samet, "Fixed point results for almost generalized cyclic $(\psi, \varphi)$-weak contractive type mappings with applications," Abstract and Applied Analysis, vol. 2012, Article ID 917831, 17 pages, 2012.

[23] E. Karapinar and K. Sadarangani, "Fixed point theory for cyclic weak $(\varphi, \psi)$-contraction," Fixed Point Theory and Applications, vol. 2011, article 69, 2011.

[24] E. Karapınar, "Fixed point theory for cyclic weak $\varphi$ contraction," Applied Mathematics Letters, vol. 24, no. 6, pp. 822-825, 2011.

[25] S. Karpagam and S. Agrawal, "Best proximity point theorems for $p$-cyclic Meir-Keeler contractions," Fixed Point Theory and Applications, vol. 2009, Article ID 197308, 9 pages, 2009.

[26] M. Păcurar, "Fixed point theory for cyclic Berinde operators," Fixed Point Theory, vol. 12, no. 2, pp. 419-428, 2011.

[27] M. Păcurar and I. A. Rus, "Fixed point theory for cyclic $\psi$ contractions," Nonlinear Analysis. Theory, Methods \& Applications, vol. 72, no. 3-4, pp. 1181-1187, 2010.

[28] M. A. Petric, "Some results concerning cyclical contractive mappings," General Mathematics, vol. 18, no. 4, pp. 213-226, 2010.

[29] M. A. Petric and B. G. Zlatanov, Fixed Point Theorems of Kannan Type for Cyclical Contractive Conditions, University Press "Paisii Hilendarski”, Plovdiv, Bulgaria, 2010.

[30] I. A. Rus, "Cyclic representations and fixed points," Annals of the Tiberiu Popoviciu Seminar of Functional Equations, Approximation and Convexity, vol. 3, pp. 171-178, 2005. 


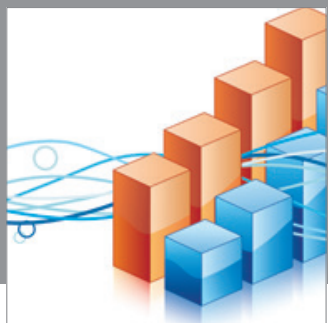

Advances in

Operations Research

mansans

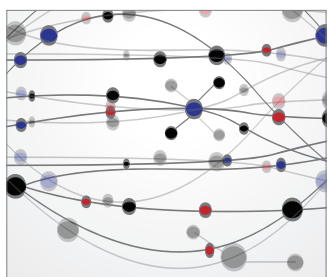

The Scientific World Journal
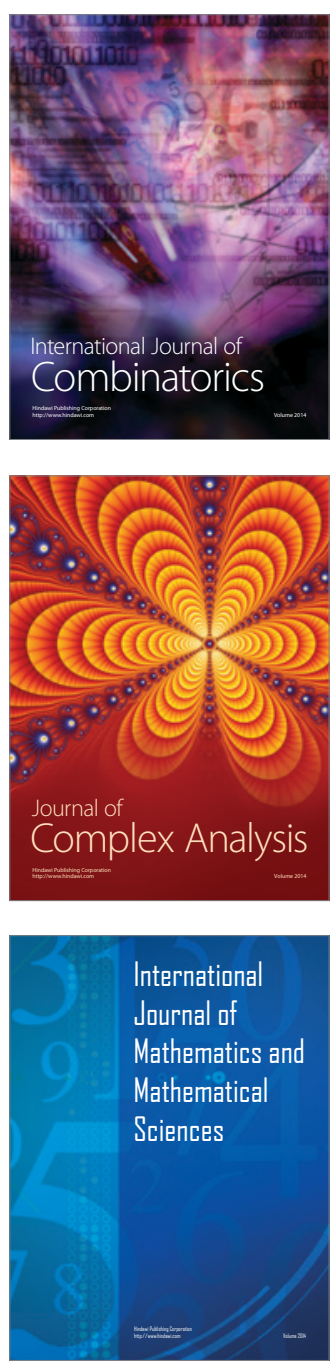
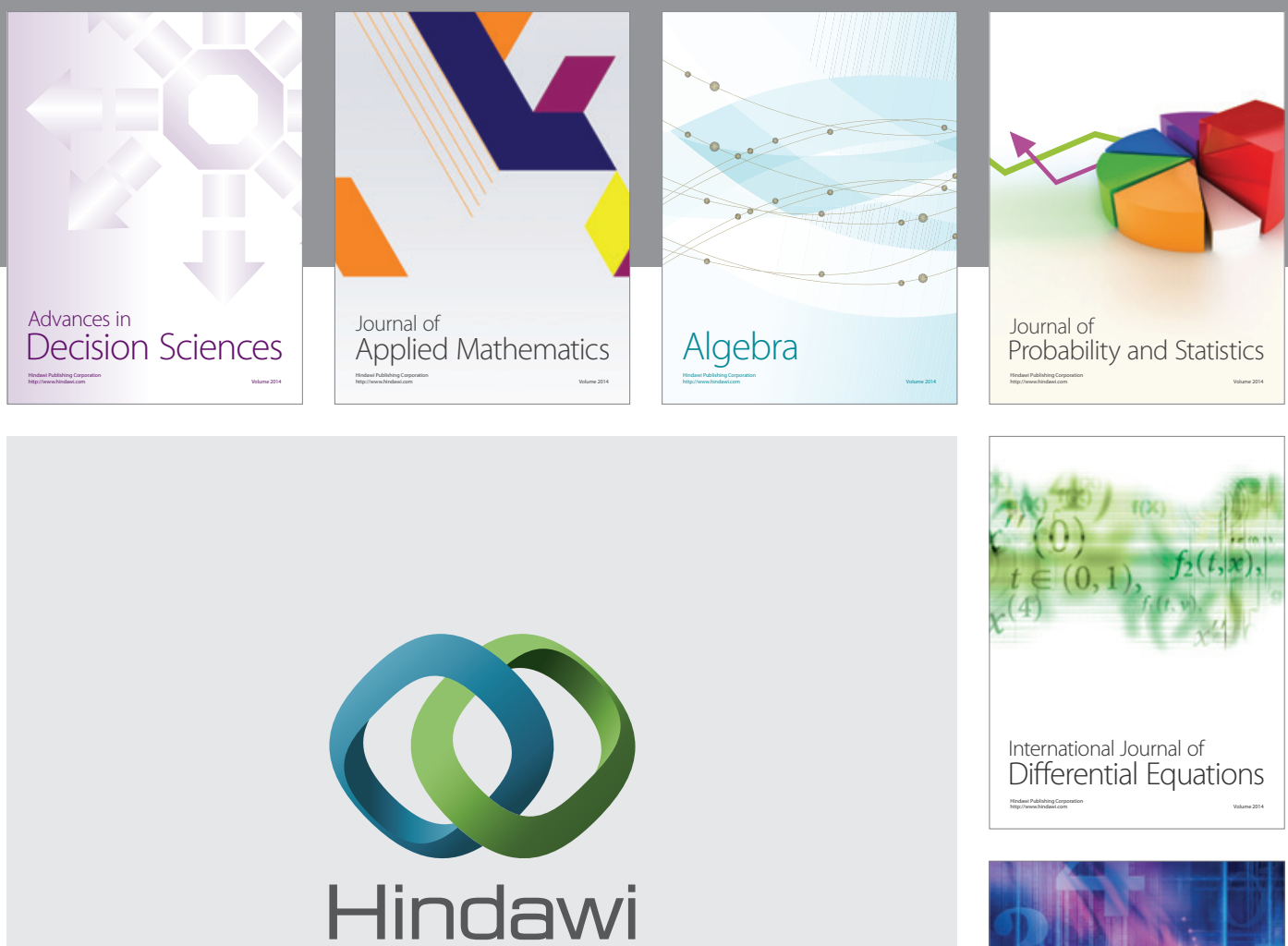

Submit your manuscripts at http://www.hindawi.com
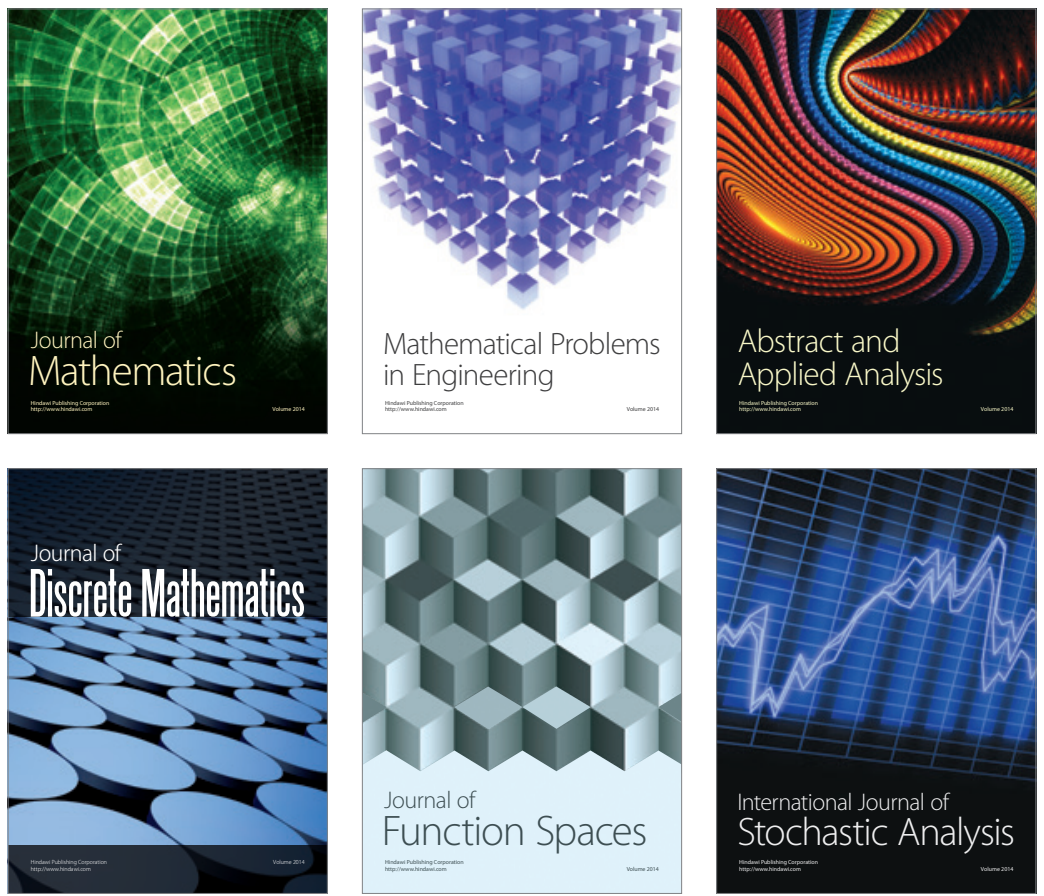

Journal of

Function Spaces

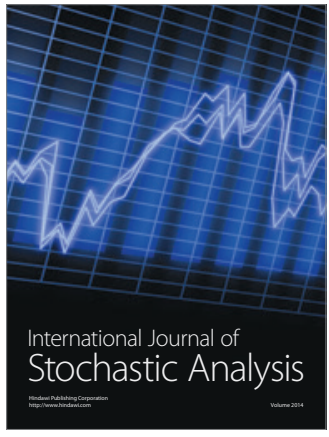

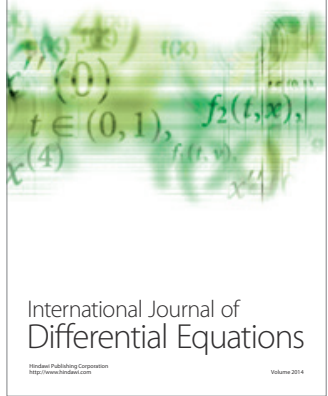
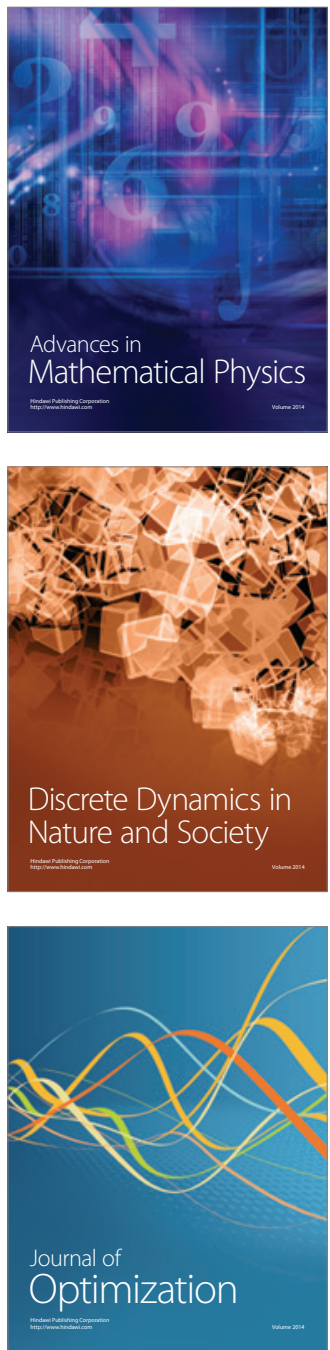